\title{
Diode-Pumped Garnet Crystal Waveguide Structures Fabricated by Pulsed Laser Deposition
}

\author{
T. C. May-Smith, J. Wang, J. I. Mackenzie, D. P. Shepherd, R. W. Eason \\ Optoelectronics Research Centre, University of Southampton, Highfield, Southampton, SO17 1BJ, UK. \\ E-mail:tcms@orc.soton.ac.uk
}

\begin{abstract}
We report progress with diode-pumping of garnet crystal waveguide structures fabricated by PLD. Lasing has been achieved in a single-layer Nd:GGG film, and a four-layer structure with a Nd:GSGG core is currently undergoing laser trials.
\end{abstract}

OCIS codes: (140.3530) Lasers, neodymium; (310.1860) Deposition and fabrication

Planar waveguide laser structures suitable for diode-pumping present an efficient means of brightness enhancement and wavelength manipulation. The planar waveguide geometry is well suited to diode-pumping, and in particular, side-pumping offers the advantage of performing lasing, pumping and thermal management each on a unique axis. Single-layer devices greater than $50 \mu \mathrm{m}$ in thickness can be utilised with high power diode-stacks with good coupling efficiency $(>80 \%)$ [1], however the use of such large thicknesses results in an undesirable highly multimode output. The use of multilayered structures, as have similarly been successfully applied to optical fibres, provides the opportunity to pump with high efficiency into thick cladding layers whilst obtaining single-mode lasing from the smaller inner core [2].

Pulsed laser deposition (PLD) is an ideal fabrication technique for making custom designed planar waveguide structures, and the use of thick layers and cladding layers has the secondary advantage of reducing the detrimental effect of particulates [3], which can otherwise be complicated to avoid. The availability of a range of different garnets with slightly different refractive indices makes it possible to produce multilayer waveguides with custom refractive index profiles. Cores based on high index garnets such as GGG or GSGG on a YAG substrate allow the high numerical apertures that are required for efficient diode-pumping to be achieved.

A single-layer Nd:GGG film and a four-layer structure consisting of a Nd:GSGG core layer, YGG cladding layers and a YAG capping layer have been fabricated using a PLD system that has been described in more detail in a previous publication [4]. Both structures were deposited on YAG (100) substrates using multiple sequential deposition runs, and in the case of the single-layer film, these were used to build up a thickness greater than $70 \mu \mathrm{m}$ so that the domed surface profile could be polished back to a more ideal planar structure. In the case of the fourlayer structure, shorter deposition runs were used with different targets to build up the resulting multilayer structure that is shown by an SEM micrograph in Figure 1.

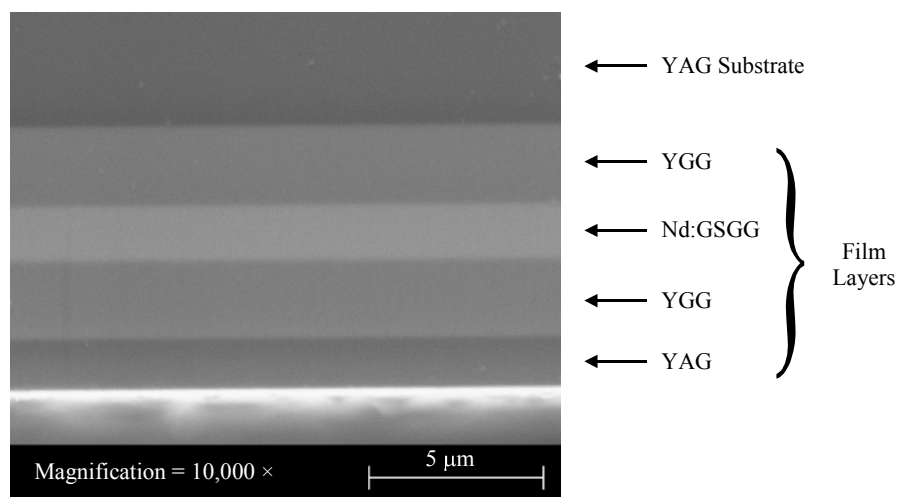

Fig. 1. SEM micrograph of a polished end face of a four-layer film structure consisting of a Nd:GSGG core, YGG cladding layers, a YAG capping layer and a YAG substrate.

The $50 \mu \mathrm{m}$ thick Nd:GGG film was further processed to make a device and has undergone optical characterisation and laser trials. Polishing of the upper face and ends resulted in a film that was $50 \mu \mathrm{m}$ in thickness and $7 \mathrm{~mm}$ in length. We have observed a significant amount of strain in films over $40 \mu \mathrm{m}$ in thickness and this strain caused cracking to occur whilst polishing, and prevented all four sides from being adequately polished, leaving only the option of end-pumping. We believe the strain is caused by a Ga deficiency in the film which forces some of the Gd ions to occupy octahedral sites in the crystal that would normally only be occupied by Ga ions. The waveguide was 
mounted on a water cooled heat-sink and a laser cavity was formed by butt-coupling mirrors to both ends of the waveguide with a thin layer of fluorinert liquid. End-pumping was performed with a three bar diode-stack capable of emitting $\sim 180 \mathrm{~W}$ at a wavelength around $808 \mathrm{~nm}$. The diodes were temperature-tuned to an optimal wavelength for absorption in the waveguide and a combination of three lenses was used to focus into the waveguide.

The absorption and fluorescence spectra for the $50 \mu \mathrm{m}$ thick film were similar to bulk Nd:GGG but slightly broadened. This has been observed in our previous films [4], and is thought to be a consequence of the Ga deficiency; the occupation of some octahedral sites by Gd results in neodymium ions experiencing slightly varied crystal fields. EDX analysis of films grown with the same conditions revealed the films to have a composition of $\mathrm{Gd}_{3.5 \pm 0.1} \mathrm{Ga}_{4.5 \pm 0.1} \mathrm{O}_{12}$ (the concentration of $\mathrm{Nd}^{3+}$ was too small to be detected). XRD analysis has shown that this level of deficiency is tolerated by the growing crystal and highly textured crystal has grown with a lattice constant slightly larger than stoichiometric GGG (due to the higher than normal concentration of the larger Gd ion).

Lasing was observed centred at wavelengths of $1060.6 \mathrm{~nm}$ and $1061.3 \mathrm{~nm}$, and was spectrally unstable; the strongest output was always centred on a wavelength of $1060.6 \mathrm{~nm}$. A minimum laser threshold of $7.4 \mathrm{~W}$ absorbed power was observed with a $\sim 2 \%$ transmittance output coupling mirror and a maximum output power of $4.0 \mathrm{~W}$ was observed with a $\sim 13 \%$ transmittance output coupling mirror. This is believed to be the highest output power ever observed from a waveguide laser device fabricated by PLD. The end-pumping configuration was limited to a maximum of $100 \mathrm{~W}$ of the $\sim 180 \mathrm{~W}$ of incident pump power available due to difficulties with prolonged mirror contact at such high incident power levels. A slope efficiency of $11.6 \%$ was obtained using a $\sim 13 \%$ transmittance output coupling mirror as shown in Figure 2. This was lower than that obtained with Ti:sapphire pumping and is thought to be due to poor overlap of the pump and laser modes. An upper limit estimate of $0.8 \mathrm{dBcm}^{-1}$ has been found for the propagation loss using the Findlay-Clay technique. This is a higher value than was obtained using Ti:sapphire pumping and is thought to represent an average loss across the whole film.

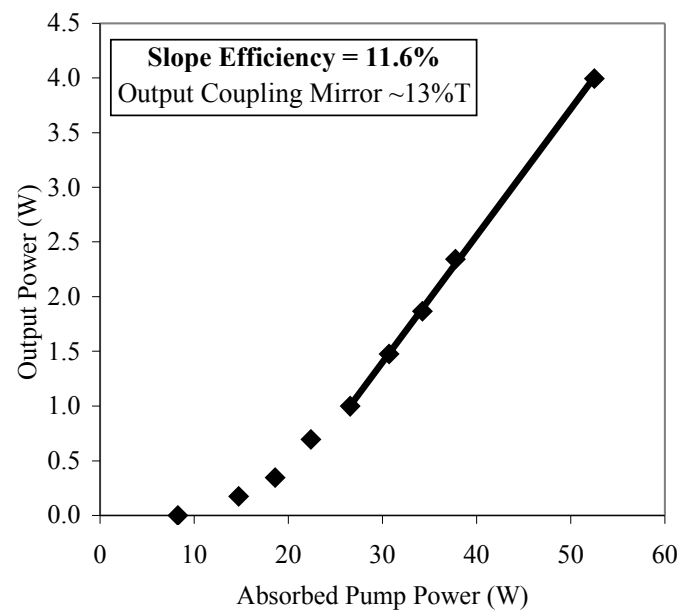

Fig. 2. Slope efficiency of a $50 \mu \mathrm{m}$ thick Nd:GGG film pumped by diode-stack using a $\sim 13 \%$ transmittance output coupler.

The results from these experiments so far have shown that PLD waveguides are capable of being pumped with very high incident power levels without fracturing. Although the end-pumping configuration used for the $50 \mu \mathrm{m}$ thick film was not ideal, it is promising that operation of the device was successful and it is thought that it will be possible to make higher performance devices once the problem of Ga deficiency has been addressed.

The four-layer structure has been polished and is currently undergoing laser trial experiments with a diode-bar pumping arrangement. The results of these cladding-pumping experiments will also be reported.

\section{References:}

[1] J. Wang, J. I. Mackenzie, and D. P. Shepherd, "A diode-stack side-pumped waveguide laser," 25th Annual Conference on Lasers and Electro-Optics, CLEO/QELS and PhAST Technical Digest, Paper Code CThI6, Baltimore, MD , May (2005).

[2] D. P. Shepherd, S. J. Hettrick, C. Li, J. I. Mackenzie, R. J. Beach, S. C. Mitchell, and H. E. Meissner, "High-power planar dielectric waveguide lasers," J. Phys. D.: Appl. Phys. 34, 2420-2432 (2001).

[3] S. J. Barrington, T. Bhutta, D. P. Shepherd, and R. W. Eason, "The effect of particulate density on performance of $\mathrm{Nd} \mathrm{Gd}_{3} \mathrm{Ga}_{5} \mathrm{O}_{12} \mathrm{waveguide}$ lasers grown by pulsed laser deposition," Opt. Commun. 185, 145-152 (2000).

[4] T. C. May-Smith, C. Grivas, D. P. Shepherd, R. W. Eason, and M. J. F. Healy, "Thick film growth of high optical quality low loss $\left(0.1 \mathrm{dBcm}^{-1}\right) \mathrm{Nd}: \mathrm{Gd}_{3} \mathrm{Ga}_{5} \mathrm{O}_{12}$ on $\mathrm{Y}_{3} \mathrm{Al}_{5} \mathrm{O}_{12}$ by pulsed laser deposition," Appl. Surf. Sci. 223, 361-371 (2004). 